\title{
Comparison of Magnetic and Non-Magnetic Nanoparticles as Sonosensitizers in Ultrasonic Hyperthermia
}

\author{
K. Kaczmarek, R. Bielas*, M. Siluk, T. Hornowski and A. Józefczak \\ Institute of Acoustics, Faculty of Physics, Adam Mickiewicz University in Poznań, \\ Uniwersytetu Poznańskiego 2, 61-614 Poznań, Poland
}

\begin{abstract}
Nanoparticles have attracted a great interest in scientific world because of the new applications they offer. They are commonly used in medical procedures such as hyperthermia and thermal ablation. Here, we propose, using magnetic and non-magnetic nanoparticles, as sonosensitizers which are the materials that improve the efficiency of heating induced by ultrasound. A comparison between various types of nanomaterials, such as magnetite, silicon dioxide, and Laponite nanoparticles, was done. The results show that both magnetic and non-magnetic nanomaterials can be utilized in ultrasonic heating. However, magnetite nanoparticles without surface modification can strongly interact with each other and are prone to agglomeration that can deteriorate thermal effect in tissuemimicking phantoms.
\end{abstract}

DOI: 10.12693/APhysPolA.137.653

PACS/topics: magnetic nanoparticles, ultrasonic hyperthermia, sonosensitizers, specific absorption rate

\section{Introduction}

Ultrasonic waves are often used to induce a temperature rise and corresponding biological effects in tissues. These effects depend on the temperature elevation in the heated region of a body. Mainly, we can distinguish ultrasonic hyperthermia, which can be helpful in cancer treatment in combination with chemo- or radiotherapy, and ultrasonic thermal ablation, for which the high temperature can lead to direct damage of a pathological tissue [1].

Regardless of designated temperature rise, the efficiency of ultrasonic heating can be improved by using additional materials called sonosensitizers. They increase the absorption of ultrasound in the medium and, as a result, heat generated there. Various types of materials are reported to be used as sonosensitizers, e.g., gold and magnetite [2], porous silica [3], or graphene oxide [4]. They have potential application in medicine, similar to Laponite clays nanodiscs that are not toxic and have found the applications in pharmaceutical and food industry [5].

The ongoing challenge is to enhance the therapeutic effect of hyperthermia without increasing the sonication time or the applied ultrasound power. A possible solution can be a combination of different modalities in one therapy procedure. For this purpose the magnetic nanoparticles can be used, as they can act as both sonosensitizers and heat sources in magnetic hyperthermia treatment $[6,7]$. In case of potential medical application the toxicity and biocompatibility of nanoparticles should be considered. These strongly depend on concentration of materials used. It can be stated that silicon dioxide, Laponite, and magnetite nanoparticles can be used in

*corresponding author; e-mail: rafal.bielas@amu.edu.pl such applications $[5,8]$. In this study, we investigated the influence of the magnetic and non-magnetic nanoparticles on effectiveness of ultrasonic hyperthermia. The research was performed in agar-based tissue-mimicking phantoms to imitate the conditions of human tissues.

\section{Experimental procedures}

In the experiments we used two types of tissuemimicking agar phantoms with and without nanoparticles. The former were prepared by mixing aqueous solution of agar powder with nanoparticles using ultrasonic homogenizer (Sonoplus HD 3100, Bandelin) that operate at frequency of $20 \mathrm{kHz}$ with acoustic intensity of about $17 \mathrm{~W} / \mathrm{cm}^{2}$. This was enough to obtain homogeneous samples with well-dispersed nanoparticles. For ultrasonic attenuation measurements the samples were prepared with different mass concentrations of sonosensitizers (from 0.4 to $4 \%$ ). The agar powder concentration was $4 \%$. The magnetite nanoparticles (50-100 nm in size) and silicon dioxide nanoparticles ( $5-15 \mathrm{~nm}$ in size) were purchased from Sigma-Aldrich Co. The magnetite nanoparticles characterized by SQUID magnetometer exhibited small magnetic hysteresis and saturation magnetization of $91 \mathrm{emu} / \mathrm{g}$ [9]. The Laponite nanoparticles were purchased from Laponite Inc. They were trioctahedral clay particles with lithium substituting for magnesium in the octahedral layers. They are usually in the form of nanosize discs $(25 \mathrm{~nm})$ [5]. All the nanoparticles were purchased in the form of powder.

In order to measure the ultrasonic attenuation coefficients, the samples were placed in distilled water between two ultrasonic transducers - transmitter and receiver (Optel Ltd.) that worked in transmission mode at frequency of $5 \mathrm{MHz}$. The attenuation coefficient was calculated on the basis of a change in amplitudes of signal registered on a receiver after transmission through samples of different thickness. For calorimetric experiments 
agar phantom was immersed in distilled water to ensure the acoustic impedance matching. Commercially used ultrasonic transducer (Sonaris S, Astar) enabled sonication of $1 \mathrm{MHz}$ or $3.5 \mathrm{MHz}$ continuous ultrasonic wave at intensity of $2.5 \mathrm{~W} / \mathrm{cm}^{2}[9]$. Temperature rise induced by the ultrasound was measured for $180 \mathrm{~s}$ by thermometer (accuracy of $0.1^{\circ} \mathrm{C}$ ) with $\mathrm{K} / \mathrm{J} / \mathrm{T}$ type thermocouple (AZ Instruments, model AZ 8852) placed in the middle of sample.

\section{Results}

The heat generated in the sonicated medium e.g., in tissue-mimicking phantom depends on the attenuation of ultrasonic waves. At first we measured the attenuation coefficient for the phantoms doped with different nanoparticles. The results are presented in Fig. 1.

As one can see, the attenuation of ultrasound in phantoms strongly depends on the concentration of nanoparticles. The biggest attenuation is observed in case of phantoms with magnetite nanoparticles, so they are expected to show the highest heating effect. On the other hand, the smallest attenuation occurs for the phantoms containing the Laponite discs. Although they were reported in the literature to tend to form 3D structures [10] that should result in the increase of ultrasound attenuation, our study seems to not support this finding. It is possible that gel matrix prevents such scenario. The measurements of hyperthermic effect were carried out in the phantoms with the $4 \%$ concentration of nanoparticles because the most significant change in ultrasonic attenuation was observed for this concentration.

Figure 2 shows the temperature rise vs. sonication time for different nanoparticles. It is clear that within the measurement time $(180 \mathrm{~s})$ the temperature of pure agar phantoms increased by $10^{\circ} \mathrm{C}$ above room temperature whereas temperature rise for phantoms with nanoparticles was significantly higher.

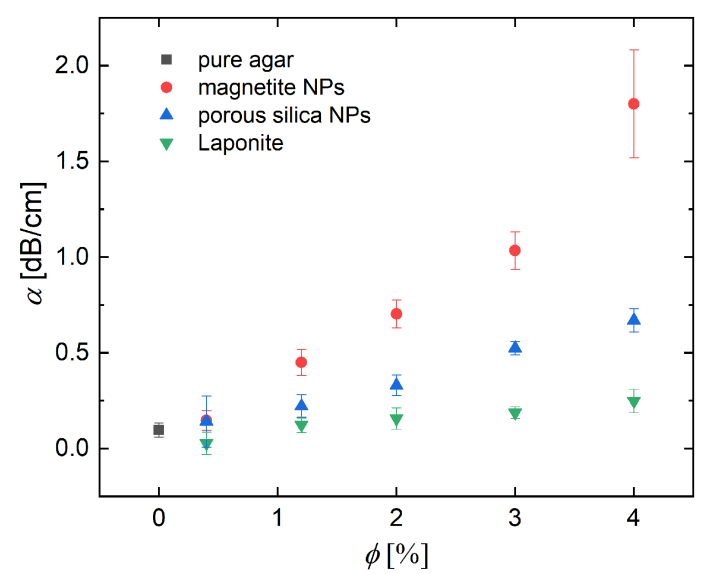

Fig. 1. Ultrasonic attenuation coefficient $\alpha$ in the function of mass concentration $\phi$ of different types of particles. Black square refers to the attenuation for agar phantom without nanoparticles.
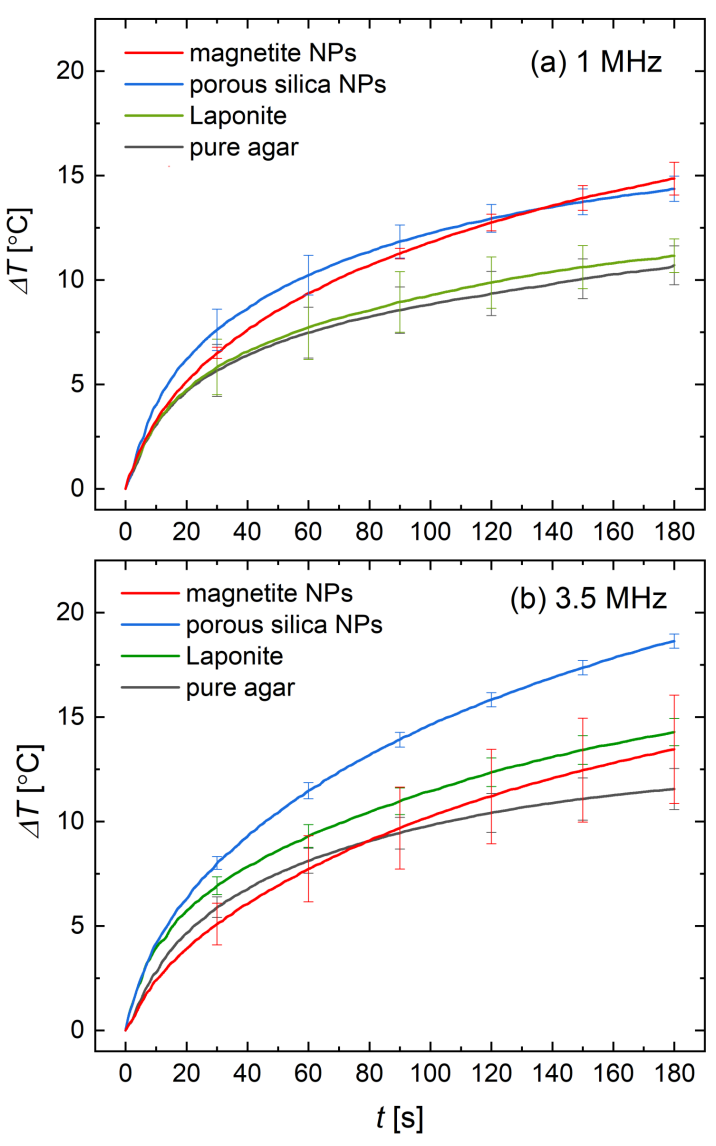

Fig. 2. The temperature rise $\Delta T$ vs. sonication time $t$ for (a) $1 \mathrm{MHz}$ and (b) $3.5 \mathrm{MHz}$ frequency of ultrasonic waves.

Figure 3 shows the efficiency of ultrasonic heating expressed by the change in temperature, $\Delta T$, after $180 \mathrm{~s}$ of sonication (averaged over at least 10 measurements) for different sonosensitizers. For $1 \mathrm{MHz}-$ ultrasound the maximal temperature rise agrees well with the predictions based on the assumption that the bigger ultrasound attenuation means better heating efficiency. The addition of solid nanoparticles increases the ultrasound attenuation in phantom, leading to the better heating efficiency. The usage of the most attenuating phantoms (with magnetite nanoparticles - in this experiment) resulted in the highest temperature rise.

However, this is not the case for $3.5 \mathrm{MHz}-$ ultrasound. One can see that the magnetite nanoparticles significantly deviate from the trend of the phantoms characterized by the bigger ultrasonic attenuation exhibiting better heat efficiency. The magnetic particles used in our experiments were in the form of powder and did not have modified surface to prevent agglomeration due to magnetic interaction between particles. On the other hand, the agglomeration leads to the formation of bigger structures that strongly scatter ultrasound of higher frequency. The intense scattering means that less of ultrasound energy is converted into heat. It is worth pointing 


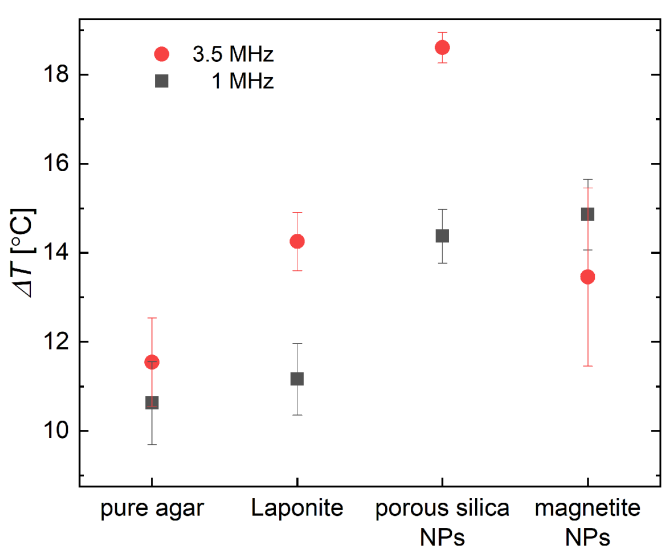

Fig. 3. Temperature elevation in tissue-mimicking phantoms for different frequencies of ultrasonic waves.

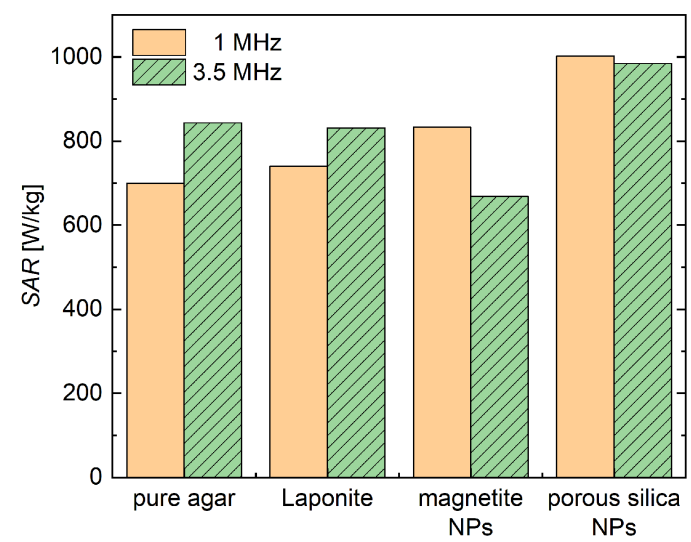

Fig. 4. Specific absorption rate (SAR) values for various nanoparticles and $1 \mathrm{MHz}$ and $3.5 \mathrm{MHz}-$ ultrasound. SAR values were calculated by fitting the Box-Lucas equation to the data shown in Fig. 2.

out that in the case of magnetic particles coated with surfactants and suspended in carrier fluid there is a good mechanism of steric repulsion that prevents the agglomeration of particles [11]. For non-magnetic nanoparticles there are no strong interactions between them. The heat generated in phantoms doped with non-magnetic material agrees with the assumption that bigger attenuation (absorption) means better heat efficiency.

From the heating rate curves presented in Fig. 2 the specific absorption rate (SAR) was calculated using the Box-Lucas equation [12]:

$$
\Delta T(t)=\Delta T_{\max }(1-\exp (-t / \tau)),
$$

where $\Delta T_{\max }$ is the maximum temperature rise and $\tau$ is a constant. The results are presented in Fig. 4, where SAR is expressed as

$$
S A R=c_{p} \frac{\mathrm{d} T}{\mathrm{~d} t} .
$$

In Eq. (2), $c_{p}$ is the specific heat of the sample and $\frac{\mathrm{d} T}{\mathrm{~d} t}$ is the rate of temperature rise. $\Delta T_{\max }$ and $\tau$ are derived from fitting experimental curves to the Box-Lucas equation, $\frac{\mathrm{d} T}{\mathrm{~d} t}=\frac{\Delta T_{\max }}{\tau}$.
The calculated SAR values prove the deterioration of heating efficiency in phantoms doped with magnetite nanoparticles, especially for higher frequency of ultrasound (Fig. 4). The results indicate that despite having the highest attenuation coefficient magnetite nanoparticles have less SAR value in comparison to silica nanoparticles. The highest efficiency of thermal energy transfer was achieved for agar phantoms doped with porous silica nanoparticles.

\section{Conclusions}

In this research we showed that sonosensitizers improve generation of heat in tissue-mimicking phantoms due to the increase in ultrasonic attenuation. This effect strongly depends on the type of nanomaterial and its physical properties. Mainly, the more attenuating the phantom is, the better is the efficiency of ultrasonic hyperthermia. However, this conclusion can be true only for non-magnetic materials (porous silica and Laponite) where the interparticle interaction can be neglected. In magnetic materials there is strong dipole-dipole attracting force which can cause the formation of the aggregates leading to the strong scattering of ultrasound. This effect generally deteriorates the temperature rise, especially for higher frequency ultrasound [9]. The presence of particles clusters explains also a significantly higher standard deviation of the hyperthermia measurements in phantoms with magnetite nanoparticles, much higher in comparison to the other types of nanoparticles. Therefore, it is important to utilize surface modification of magnetic particles minimizing the effect of magnetic interactions, especially for applications like magneto-ultrasonic hyperthermia [7].

\section{Acknowledgments}

This research was funded by the grants 2015/17/ B/ST7/03566 (OPUS) and 2017/27/N/ST7/00201 (PRELUDIUM) of the Polish National Science Center. We wish to thank Mr. S. Porzak for his experimental help during attenuation measurements.

\section{References}

[1] H.P. Kok, A.N.T.J. Kotte, J. Crezee, Int. J. Hyper. 33, 593 (2017).

[2] S.B. Devarakonda, M.R. Myers, R.K. Banerjee, J. Biomech. Eng. 140, 081003 (2018).

[3] A.P. Sviridov, V.G. Andreev, E.M. Ivanova, L.A. Osminkina, K.P. Tamarov, V.Yu. Timoshenko, Appl. Phys. Lett. 103, 193110 (2013).

[4] J. Beik, Z. Abed, A. Shakeri-Zadeh, M. Nourbakhsh, M.B. Shiran, Physica E 81, 308 (2016).

[5] H. Tomás, C.S. Alves, J. Rodrigues, Nanomedicine 14, 2407 (2018).

[6] S.A.R. Dibaji, M.F. Al-Rjoub, M.R. Myers, R.K. Banerjee, J. Nanotechnol. Eng. Med. 4, 040902 (2014). 
[7] K. Kaczmarek, T. Hornowski, I. Antal, M. Timko, A. Józefczak, J. Magn. Magn. Mater. 474, 400 (2019).

[8] G. Crisponi, V.M. Nurchi, J.I. Lachowicz, M. Peana, S. Medici, M.A. Zoroddu, in: Antimicrobial Nanoarchitectonics, Ed. A.M. Grumezescu, Elsevier, Amsterdam 2017, p. 511.

[9] K. Kaczmarek, T. Hornowski, R. Bielas, D. Żak, M. Timko, A. Józefczak, Acta Phys. Pol. A 133, 716 (2018).
[10] S. Ali, R. Bandyopadhyay, Langmuir 29, 12663 (2013).

[11] C. Barrera, A.P. Herrera, C. Rinaldi, J. Coll. Interface Sci. 329, 107 (2009).

[12] U.M. Engelmann, J. Seifert, B. Mues, S. Roitsch, C. Ménager, A.M. Schmidt, I. Slabu, J. Magn. Magn. Mater. 471, 486 (2019). 